\title{
Pendeo-epitaxial Growth and Characterization of GaN and related Materials on 6H-SiC(0001) and $\mathrm{Si}(111)$ Substrates
}

Robert F. Davis, T. Gehrke, K.J. Linthicum ${ }^{+}$, T. S. Zheleva, P. Rajagopal ${ }^{+}$, C. A.

Zorman* and M. Mehregany*

Department of Materials Science and Engineering, North Carolina State University, Raleigh, NC 27695; *Department of Electrical and Computer Engineering, Case Western Reserve University, Cleveland, $\mathrm{OH} 44106$

\begin{abstract}
Discrete and coalesced monocrystalline $\mathrm{GaN}$ and $\mathrm{Al}_{\mathrm{x}} \mathrm{Ga}_{1-\mathrm{x}} \mathrm{N}$ layers grown via Pendeoepitaxy (PE) [1] originated from side walls of $\mathrm{GaN}$ seed structures containing $\mathrm{SiN}_{\mathrm{x}}$ top masks have been grown via organometallic vapor phase deposition on GaN/AlN/6H$\mathrm{SiC}(0001)$ and $\mathrm{GaN}(0001) / \mathrm{AlN}(0001) / 3 \mathrm{C}-\mathrm{SiC}(111) / \mathrm{Si}(111)$ substrates. Scanning and transmission electron microscopies were used to evaluate the external microstructures and the distribution of dislocations, respectively. The dislocation densities in the PE grown films was reduced by at least five orders of magnitude relative to the initial GaN seed layers. Tilting in the coalesced $\mathrm{GaN}$ epilayers was observed via X-ray diffraction. A tilt of $0.2^{\circ}$ was confined to areas of mask overgrowth; however, no tilting was observed in the material suspended above the $\mathrm{SiC}$ substrate. The strong, low-temperature PL band-edge peak at $3.45 \mathrm{eV}$ with a FWHM of $17 \mathrm{meV}$ was comparable to that observed in PE GaN films grown on $6 \mathrm{H}-\mathrm{SiC}(0001)$. The band-edge in the $\mathrm{GaN}$ grown on $\mathrm{AlN}(0001) / \mathrm{SiC}(111) \mathrm{Si}(111)$ substrates was shifted to a lower energy by $10 \mathrm{meV}$, indicative of a greater tensile stress.
\end{abstract}

\section{INTRODUCTION}

It has been a necessity for investigators in the III-nitride community to grow films of $\mathrm{GaN}$ and related nitride materials using heteroepitaxial growth routes because of the dearth of bulk substrates of these materials. This results in films containing dislocation densities of $10^{8}-10^{10} \mathrm{~cm}^{-2}$ because of the mismatches in the lattice parameters and the coefficients of thermal expansion between the buffer layer and the film and/or the buffer layer and the substrate. These high concentrations of dislocations may also limit the performance of devices.

Several groups [2-10], including the present authors, have conducted research regarding selective area growth (SAG) and lateral epitaxial overgrowth (LEO) techniques for GaN deposition, specifically to reduce significantly the dislocation density. Increased emphasis in this research topic was fueled in part by the announcement by Nakamura, et al. [11-13] of the dramatic increase in projected lifetime of their GaN- based blue lightemitting laser diodes fabricated on LEO material. Using these approaches, researchers have been able to grow GaN films containing dislocation densities of $\approx 10^{5} \mathrm{~cm}^{-2}$ in the areas of overgrowth. 
${ }^{+}$Now with: Nitronex Corporation, Raleigh, NC 27606

However, to benefit from this reduction in defects, the placement of devices incorporating LEO technology is limited and confined to regions on the final GaN device layer that are located on the overgrown regions.

Recently we have pioneered a new approach to selective epitaxy of $\mathrm{GaN}$ and $\mathrm{Al}_{\mathrm{x}} \mathrm{Ga}_{1-\mathrm{x}} \mathrm{N}$ layers, namely, pendeo- (from the Latin: to hang or be suspended) epitaxy (PE) $[1,14-19]$ as a promising new process route leading to a single, continuous, large area layer; multilayer heterostructures or discrete platforms of these materials. It incorporates mechanisms of growth exploited by the conventional LEO process by using an amorphous mask to prevent vertical propagation of threading dislocations; however, it extends beyond the conventional LEO approach to employ the substrate itself as a pseudo-mask. This unconventional approach differs from LEO in that growth does not initiate through open windows on the (0001) surface of the GaN seed layer; instead, it is forced to selectively begin on the sidewalls of a tailored microstructure comprised of forms previously etched into this seed layer. Continuation of the pendeo-epitaxial growth of $\mathrm{GaN}$ or the growth of the $\mathrm{Al}_{\mathrm{x}} \mathrm{Ga}_{1-\mathrm{x}} \mathrm{N}$ layer until coalescence over and between these forms results in a complete layer of low defect-density $\mathrm{GaN}_{\text {or }} \mathrm{Al}_{\mathrm{x}} \mathrm{Ga}_{1-\mathrm{x}} \mathrm{N}$. This is accomplished in one $(\mathrm{GaN})$, two $\left(\mathrm{Al}_{\mathrm{x}} \mathrm{Ga}_{1-\mathrm{x}} \mathrm{N}\right)$ or multiple (multilayer heterostructure) re-growth steps. And the need to align devices or masks for the growth of the subsequent layers over particular areas of overgrowth is eliminated, unless deposition only in certain areas is desired.

The following sections describe the experimental parameters necessary to achieve $\mathrm{GaN}$ and $\mathrm{Al}_{\mathrm{x}} \mathrm{Ga}_{1-\mathrm{x}} \mathrm{N}$ films via $\mathrm{PE}$ on $\mathrm{SiC}(0001)$ and $\mathrm{Si}(111)$ substrates. The microstructural and optical evidence obtained for the resulting films is also described, discussed and summarized.

\section{EXPERIMENTAL PROCEDURES}

Each pendeo-epitaxial $\mathrm{GaN}$ and $\mathrm{Al}_{\mathrm{x}} \mathrm{Ga}_{1-\mathrm{x}} \mathrm{N}$ film and the underlying $\mathrm{GaN}$ seed layer and the AlN buffer layer were grown in a cold-wall, vertical pancake style RF inductively heated metallorganic vapor phase epitaxy (MOVPE) system. Two distinct process routes were explored for growth on (i) on-axis $6 \mathrm{H}-\mathrm{SiC}(0001)$ substrates and (ii) on-axis $\mathrm{Si}(111)$ substrates. In the former, each seed layer consisted of a $1 \mu \mathrm{m}$ thick $\mathrm{GaN}$ film grown on a $100 \mathrm{~nm}$ thick AlN buffer layer previously deposited on a $6 \mathrm{H}-\mathrm{SiC}(0001)$ substrate. Details of the experimental parameters used for the growth of these two layers are given in Ref. [20]. In the growth on the Si substrates, a $1 \mu \mathrm{m}$ 3C-SiC(111) film was initially grown on a very thin $3 \mathrm{C}-\mathrm{SiC}(111)$ layer produced by conversion of the $\mathrm{Si}(111)$ surface at $1360^{\circ} \mathrm{C}$ for $90 \mathrm{~s}$ via reaction with $\mathrm{C}_{3} \mathrm{H}_{8}$ entrained in $\mathrm{H}_{2}$. The film was subsequently achieved by simultaneously decreasing the flow rate of the $\mathrm{C}_{3} \mathrm{H}_{8} / \mathrm{H}_{2}$ mixture and introducing a $\mathrm{SiH}_{4} / \mathrm{H}_{2}$ mixture. Both the conversion step and the $\mathrm{SiC}$ film deposition were achieved using a cold-wall, vertical geometry, RF inductively heated atmospheric pressure chemical vapor deposition (APCVD) reactor. Details of the experimental parameters used for the conversion step and the growth of the 3C-SiC layer are given in Ref. [21]. A $100 \mathrm{~nm}$ thick AIN buffer layer and a $1 \mu \mathrm{m} \mathrm{GaN}$ seed layer were subsequently deposited in the manner described above for the $6 \mathrm{H}-\mathrm{SiC}$ substrates. 
A $100 \mathrm{~nm}$ silicon nitride growth mask was deposited on the seed layers via plasma enhanced CVD. A nickel etch mask was subsequently deposited using e-beam evaporation. Patterning of the nickel mask layer was achieved using standard photolithography techniques. The final, tailored, microstructure consisting of seed forms was fabricated via removal of portions of the nickel etch mask via sputtering and by inductively coupled plasma (ICP) etching of portions of the silicon nitride growth mask, the GaN seed layer and the AIN buffer layer. Critical to the success of the pendeo-epitaxial growth, the etching of the seed-forms was continued completely through the exposed GaN and AlN layers and into either the $6 \mathrm{H}-\mathrm{SiC}$ substrate or the $3 \mathrm{C}-\mathrm{SiC}$ layer, thereby removing all III- nitride material from the areas between the side walls of the forms. The seed forms used in this study were raised rectangular stripes oriented along the [1 100$]$ direction, thereby providing a sequence of parallel GaN sidewalls (nominally (1120) faces). Seed form widths of 2 and $3 \mu \mathrm{ms}$ coupled with separation distances of 3 and $7 \mu \mathrm{ms}$ were employed. The remaining nickel mask that protected the seed structures during the ICP etching process was removed using a wet etch. Immediately prior to pendeo-epitaxial growth, the patterned samples were dipped in a acid solution to remove surface contaminants from the walls of the underlying GaN seed structures.

A schematic of the pendeo-epitaxial growth of $\mathrm{GaN}$ is illustrated in Figure 1. There are three primary stages associated with the pendeo-epitaxial formation of this material: (i) initiation of lateral homoepitaxy from the sidewalls of the $\mathrm{GaN}$ seed, (ii) vertical growth and (iii) lateral growth over the silicon nitride mask covering the seed structure. Pendeo-epitaxial growth of GaN was achieved within the temperature range of $1050-1100^{\circ} \mathrm{C}$ using the same pressure and V/III ratio used for the deposition of the GaN seed layer, as described above. Additional experimental details regarding the pendeoepitaxial growth of $\mathrm{GaN}$ and $\mathrm{Al}_{\mathrm{x}} \mathrm{Ga}_{1-\mathrm{x}} \mathrm{N}$ layers employing $6 \mathrm{H}-\mathrm{SiC}$ substrates are given in Refs. [14-19, 22]. The morphology and defect microstructures were investigated using scanning electron microscopy (SEM) (JEOL 6400 FE) and transmission electron microscopy (TEM) (TOPCON 0002B, $200 \mathrm{KV}$ ). Determination of the degree of tilting in the PE GaN was achieved using X-ray diffraction (XRD) (Philips X'Pert MRD X-ray diffractometer) analysis. Optical characterization was performed via photoluminescence (PL) using a He-Cd laser ( $\lambda=325 \mathrm{~nm})$.

\section{RESULTS AND DISCUSSION}

\section{Growth on $\mathrm{SiC}$ substrates}

The pendeo-epitaxial phenomenon is made possible by taking advantage of growth mechanisms identified by Zheleva et al. [8] in the conventional LEO technique and by using two additional key steps, namely, the initiation of growth from a GaN face other than the (0001) and the use of the substrate (in this case $\mathrm{SiC}$ ) as a pseudo-mask. By capping the seed-forms with a growth mask, the $\mathrm{GaN}$ was forced to grow initially and selectively only on the GaN sidewalls. Common to conventional LEO, no growth occurred on the silicon nitride mask covering the seed forms. Deposition also did not occur on the exposed $\mathrm{SiC}$ surface areas at the higher growth temperatures employed to enhance lateral growth (the pseudo-mask effect). The Ga- and N-containing species more likely either diffused along the surface or evaporated (rather than having sufficient time to 
form $\mathrm{GaN}$ nuclei) from both the silicon nitride mask and the silicon carbide substrate. The pronounced effect of this is shown in Figure 3 wherein the newly deposited GaN has grown truly suspended (pendeo) from the sidewalls of the GaN seed structure. During the second PE event (ii), vertical growth of GaN occurred from the advancing (0001) face of the laterally growing $\mathrm{GaN}$. Once the vertical growth became extended to a height greater than the silicon nitride mask, the third PE event (iii) occurred, namely, conventional LEOtype growth and eventual coalescence over the seed structure, as shown in Figure 3.

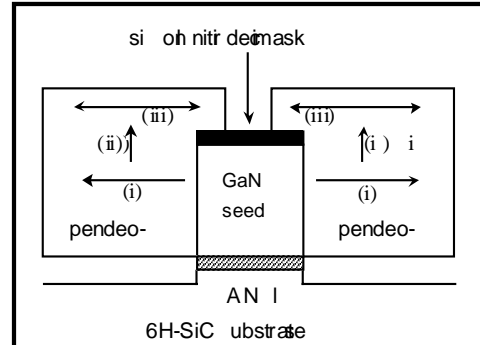

Figure 1. Schematic of pendeoepitaxial growth from $\mathrm{GaN}$ sidewalls and over a silicon nitride mask.

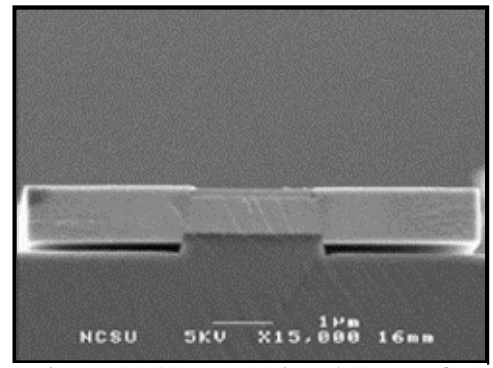

Figure 2. Cross-sectional SEM of a $\mathrm{GaN}$ pendeo-epitaxial growth structure with limited vertical growth from the seed sidewalls and no growth on the seed mask.

A cross-sectional TEM micrograph showing a typical pendeo-epitaxial growth structure is shown in Figure 4. Threading dislocations extending into the GaN seed structure, originating from the GaN/AlN and AlN/SiC interfaces are clearly visible. The silicon nitride mask acted as a barrier to the further vertical propagation of these defects into the laterally overgrown pendeo-epitaxial film. Since the newly deposited $\mathrm{GaN}$ is suspended above the $\mathrm{SiC}$ substrate, there are no vertically oriented defects associated with the mismatches in lattice parameters between GaN and AlN and between AlN and SiC. Preliminary analyses of the GaN seed/GaN PE and the AlN/GaN PE interfaces revealed evidence of the lateral propagation of the defects; however, there is yet no evidence that the defects reach the (0001) surface where device layers will be grown. As in the case of LEO, there is a significant reduction in the defect density in the regrown areas. 


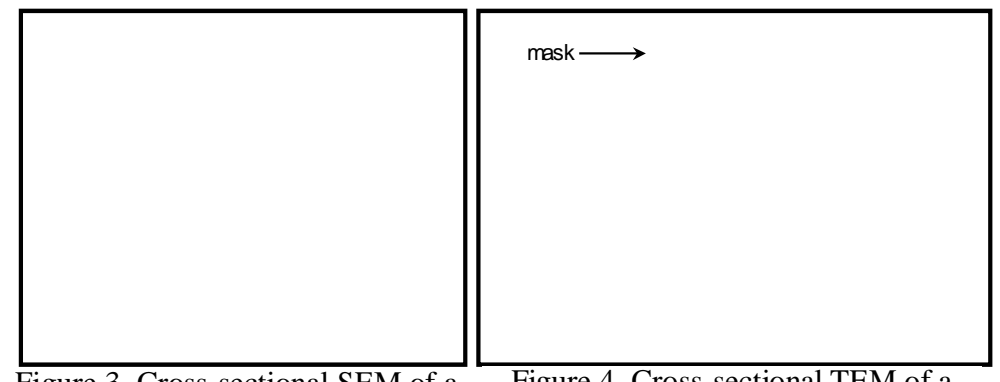

$\begin{array}{cc}\text { Figure 3. Cross-sectional SEM of a } & \text { Figure 4. Cross-sectional TEM of } \\ \text { GaN/Al Ga } \mathrm{N} \text { pendeo epitaxial } & \text { GaN pendeo-epitaxial structure }\end{array}$ growth structure showing showing confinement of threading coalescence over the seed mask. dislocations under the seed mask, and a reduction of defects in the regrowth. 
The continuation of the pendeo-epitaxial growth results in coalescence with adjacent growth fronts and the formation of a continuous layer of $\mathrm{GaN}$, as observed in Figure 5. This also results in the practical elimination of all dislocations stemming from the heteroepitaxial growth of GaN/AlN on SiC. Clearly visible in Fig. 5(a) are the voids that form when adjacent growth fronts coalesce. Optimization of the pendeo-epitaxial growth technique should eliminate these undesirable defects.
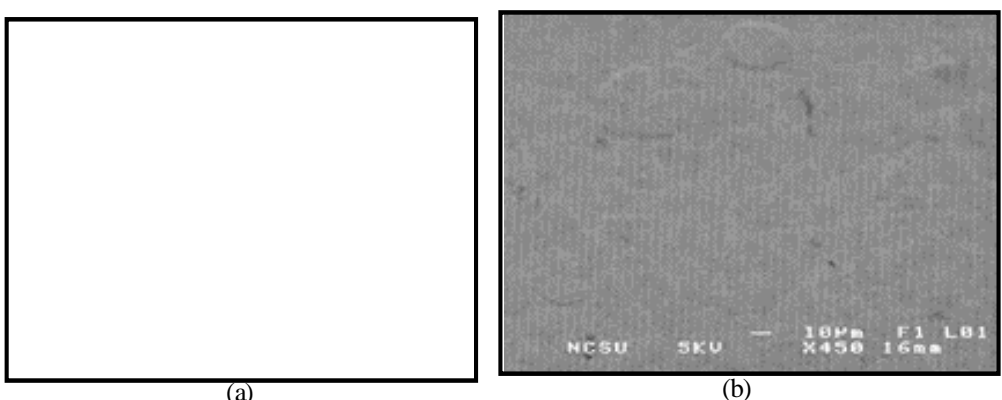

Figure 5. Micrographs taken via (a) cross-sectional SEM and (b) plan-view SEM of examples of pendeo-epitaxial growth with coalescence over and between the seed forms resulting in a single $\mathrm{GaN}$ layer.

Pendeo-epitaxial growth of individual forms and coalesced single layers of PE $\mathrm{Al}_{10} \mathrm{Ga}_{90} \mathrm{~N}$ alloys and $\mathrm{PE} \mathrm{GaN}$ as well as forms containing heterostructures of alternating layers of $\mathrm{GaN}$ and $\mathrm{Al}_{10} \mathrm{Ga}_{90} \mathrm{~N}$ have been realized in this research as shown in Figure 3. Ref. [22] is discussing in more detail the process necessary to fabricate coalesced single layers of $\mathrm{PE} \mathrm{Al}_{10} \mathrm{Ga}_{90} \mathrm{~N}$ alloys and their characterization.

\section{Growth on Silicon Substrates}

Research regarding the development of process routes leading to the growth of III-Nitride films on Si has been less relative to the number of studies concerned with growth on sapphire and SiC. This has been due in part to the three-dimensional nucleation and growth of $\mathrm{GaN}$ islands caused by the combination of significant mismatches in lattice parameters, the higher surface energy of $\mathrm{GaN}$ and the chemical reactivity of $\mathrm{Si}$ with the reactants in the growth environment. To address the above concerns we have developed a process route similar to those used for growth of $\mathrm{GaN}$ on $6 \mathrm{H}-\mathrm{SiC}(0001)$, but replaced the $6 \mathrm{H}-\mathrm{SiC}$ substrate with a $3 \mathrm{C}-\mathrm{SiC}(111)$ transition layer grown on a $\mathrm{Si}(111)$ substrate. The atomic arrangement of the (111) plane of $3 \mathrm{C}-\mathrm{SiC}$ is equivalent to the (0001) plane of $6 \mathrm{H}$ $\mathrm{SiC}$; this facilitates the sequential deposition of a high temperature $2 \mathrm{H}-\mathrm{AlN}(0001)$ buffer layer of sufficient quality for the GaN seed layer, as discussed in Reference [20]. The 3C$\mathrm{SiC}$ layer is also exposed between the $\mathrm{GaN}$ seed forms and the seed forms are capped with a silicon nitride growth mask to force the $\mathrm{GaN}$ to grow initially and selectively only on the $\mathrm{GaN}$ sidewalls in the manner described above for growth using the $6 \mathrm{H}-\mathrm{SiC}(0001)$ substrates. 
For the initial demonstrations of PE growth of GaN films on silicon, 0.5 and 2 $\mu \mathrm{m}$ thick $3 \mathrm{C}$-SiC(111) layers were deposited on $50 \mathrm{~mm}$ diameter, $250 \mu \mathrm{m}$ thick converted $\mathrm{Si}(111)$ substrates. All subsequent research described below used the $\sim 2.0 \mu \mathrm{m}$ barrier layer.

Figure 6 shows a cross-sectional SEM micrograph of a PE GaN layer grown laterally and vertically from raised GaN stripes etched in a GaN/AlN/3C-SiC/Si(111) substrate and over the silicon nitride mask atop each stripe.

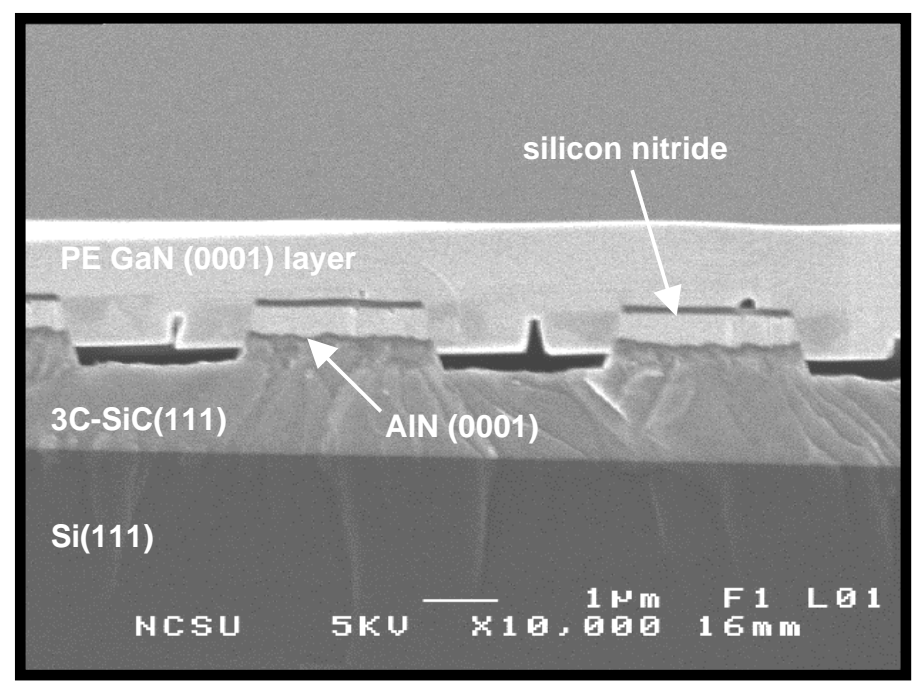

Figure 6. Cross-sectional SEM micrograph of a coalesced PE GaN epilayer deposited on a $3 \mathrm{C}-\mathrm{SiC} / \mathrm{Si}(111)$ substrate.

Tilting between adjacent growth fronts over the mask regions are commonly observed in coalesced GaN epilayers grown using the LEO technique. This phenomenon was also determined to be present in the PE GaN films.

The FWHMs of the XRD rocking curves for the (0002) reflection are indicative of the crystallographic tilt and are dependent on the crystal orientation, as shown in Figure 7. The XRD spectrum taken along the [ $1 \overline{1} 00]$, parallel to the stripes, consisted of one peak as shown in Figure 7(a), the spectrum along the [11̄20], perpendicular to the stripes, exhibited two superimposed peaks, separated by a tilt of $0.2^{\circ}$, as shown in Figure $7(\mathrm{~b})$. 


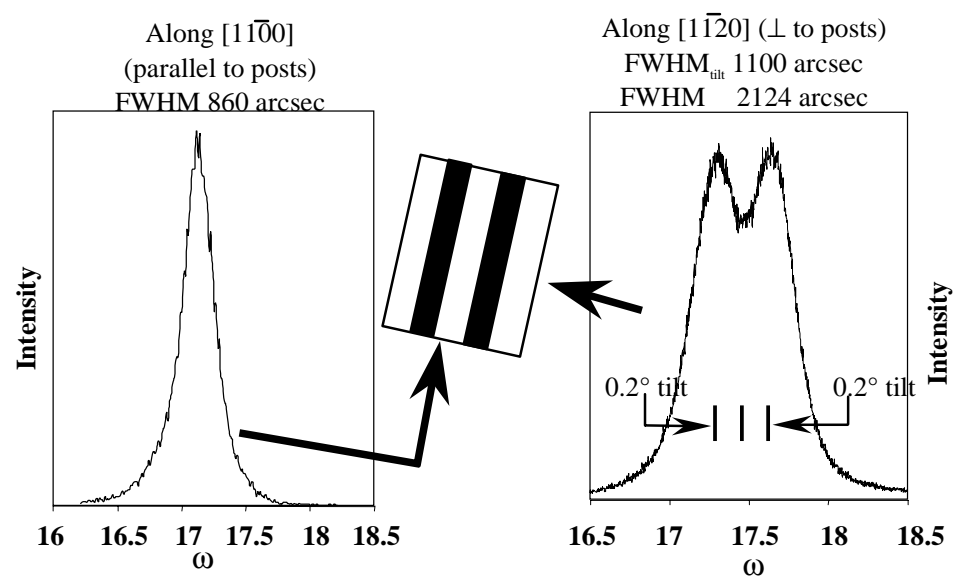

Figure 7. DCXRD analysis of the tilting in the coalesced PE films.

Unlike LEO, coalesced PE GaN epilayers contain two sets of coalesced growth fronts, namely, over the trenches and over the masks. A more sensitive method of evaluating the tilt in PE grown $\mathrm{GaN}$ is required to determine if it is present at both areas of coalescence. Selected area diffraction patterns were obtained using TEM and taken from a small area of coalescence in a trench region and near the region of coalescence over the silicon nitride mask. Analyses of these two patterns revealed no evidence of tilt in the laterally grown material over the trenches; however, significant tilt has occurred over the mask regions of the seed structures. Thus the mask has markedly influenced the crystallographic tilt in the overgrown film.

A typical room temperature PL spectrum for a PE GaN film grown on $2 \mu \mathrm{m}$

wide raised stripes, oriented in the $[1 \overline{1} 00]$ direction, spaced $3 \mu \mathrm{m}$ apart and capped with a silicon nitride mask is shown in Figure 8. The intensity of the spectrum is plotted on a linear scale. The band-edge emission peak and a deep level (yellow luminescence) emission peak were located at $363.6 \mathrm{~nm}(3.41 \mathrm{eV})$ and $\sim 570 \mathrm{~nm}$, respectively. The FWHM of the former was $109 \mathrm{meV}$.

The low temperature (14K) PL spectrum on a logarithmic intensity scale showed a strong near band-edge emission at $359 \mathrm{~nm}(3.45 \mathrm{eV})$, as shown in Figure 9 . The FWHM was $17 \mathrm{meV}$. Also included in Figure 9 is a low temperature PL spectrum of PE $\mathrm{GaN}$ grown on a GaN/AlN/6H-SiC substrate. The band-edge peak in the latter spectrum is at $358 \mathrm{~nm}(3.46 \mathrm{eV})$ with a FWHM of $16 \mathrm{meV}$.

A comparison of these two spectra indicates that the optical character of the PE $\mathrm{GaN}$ grown on the $3 \mathrm{C}-\mathrm{SiC} / \mathrm{Si}$ substrate is nearly identical to the quality of the PE GaN grown on the $6 \mathrm{H}-\mathrm{SiC}$ substrates. The band-edge of the $\mathrm{PE} \mathrm{GaN} \mathrm{grown} \mathrm{on} \mathrm{Si}$ is shifted by $10 \mathrm{meV}$ to a slightly lower energy than that of the PE GaN grown on $\mathrm{SiC}$, which is indicative of a slightly higher stress in the film of the former. As a further comparison, 
GaN(0001) films conventionally grown on AlN/6H-SiC substrates typically have a bandedge peak at $357.7 \mathrm{~nm}(3.466 \mathrm{eV})$ and a FWHM of $4 \mathrm{meV}$. 


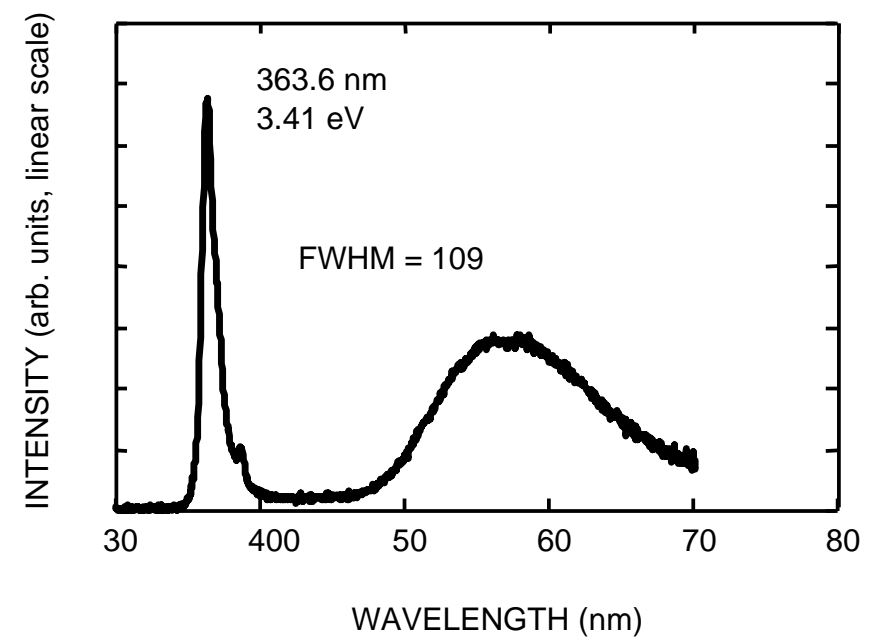

Figure 8. Room temperature photoluminescence of a coalesced layer of $\mathrm{PE} \mathrm{GaN} \mathrm{grown} \mathrm{on} \mathrm{an} \mathrm{GaN/AIN/3C-}$ $\mathrm{SiC} / \mathrm{Si}(111)$ substrate.

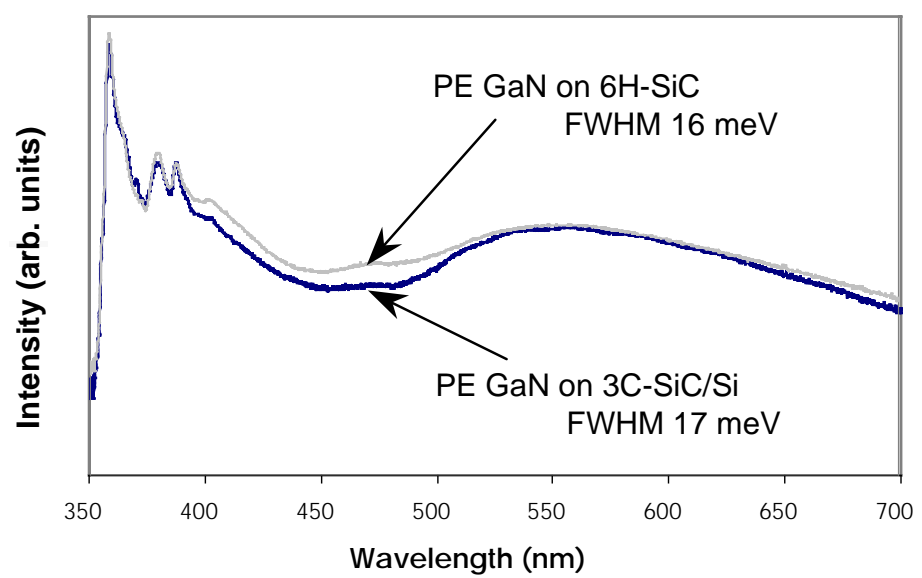

Figure 9. Comparison of low-temperature (14K) PL spectra of PE GaN grown on $\mathrm{GaN} / \mathrm{AlN} / 6 \mathrm{H}-\mathrm{SiC}$ and $\mathrm{GaN} / \mathrm{AlN} / 3 \mathrm{C}-\mathrm{SiC} / \mathrm{Si}(111)$ substrates. 


\section{SUMMARY}

Pendeo-epitaxy has been developed as an alternative and more simple approach of growing uniformly thin films with low densities of threading dislocations over the entire surface of a substrate. In particular, the growth of both discrete structures and coalesced $\mathrm{GaN}$ and $\mathrm{Al}_{\mathrm{x}} \mathrm{Ga}_{1-\mathrm{x}} \mathrm{N}$ films and multilayer heterostructures using pendeo-epitaxy on etched $\mathrm{GaN}$ seed layers previously grown on $\mathrm{AlN}(0001) / 6 \mathrm{H}-\mathrm{SiC}(0001)$ and $\operatorname{AlN}(0001) / \mathrm{SiC}(111) / \mathrm{Si}(111)$ substrates has been demonstrated. Tilting in the coalesced $\mathrm{GaN}$ epilayers of $0.2^{\circ}$ was confined to areas of mask overgrowth; no tilting was observed in the material suspended above the trenches. The strong, low-temperature PL band-edge peak at $3.45 \mathrm{eV}$ with a FWHM of $17 \mathrm{meV}$ was comparable to that observed in PE GaN films grown on $6 \mathrm{H}-\mathrm{SiC}(0001)$. The band-edge in the material on $\mathrm{Si}(111)$ was shifted by 10 $\mathrm{meV}$ to a lower energy, indicative of a greater tensile stress.

\section{ACKNOWLEDGEMENTS}

The authors acknowledge Cree Research, Inc. and Motorola for the SiC and the silicon wafers respectively. This work was supported by the Office of Naval Research under contracts N00014-98-1-0384 (Colin Wood, monitor) and N00014-98-1-0654 (John Zolper, monitor). The authors also acknowledge CWRU and the Georgia Technology Research Institute for use of their clean room facilities.

\section{REFERENCES}

1. Trademark of Nitronex Corporation, Raleigh, NC 27606

2. D. Kapolnek S. Keller, R. Vetury, R. Underwood, P. Kozodoy, S. Denbaars, and U. Mishra, Appl. Phys. Lett. 71, 1204 (1997).

3. Y. Kato, S. Kitamura, K. Hiramatsu, and N. Sawaki, J. Cryst. Growth, 144, 133 (1994).

4. O. Nam, M. Bremser, B. Ward, R. Nemanich, and R. Davis, Mat.Res. Soc. Symp. Proc., 449, 107 (1997).

5. O. Nam, M. Bremser, B. Ward, R. Nemanich, and R. Davis, Jpn.J. Appl. Phys., Part 1 36, L532 (1997).

6. Sakai, H. Sunakawa, and A. Usui, Appl. Phys. Lett., 73, 481 (1998).

7. H. Marchand, X. Wu, J. Ibbetson, P. Fini, P. Kozodoy, S. Keller, J. Speck, S. Denbaars, and U. Mishra, Appl. Phys. Lett., 73, 747 (1998).

8. T. Zheleva, O. Nam, M. Bremser, and R. Davis, Appl. Phys. Lett., 71, 2472 (1997).

9. O. Nam, T. Zheleva, M. Bremser, and R. Davis, Appl. Phys. Lett., 71, 2638 (1997).

10. H. Zhong, M. Johnson, T. McNulty, J. Brown, J. Cook Jr., J. Schetzina, Materials Internet Journal, Nitride Semiconductor Research, 3, 6, (1998). 
11. S. Nakamura, M.Senoh, S. Nagahama, N. Iwasa, T. Yamanda, T. Matsushita, H. Kiyoku, Y. Sugimoto, T. Kozaki, H. Umemoto, M. Sano, and K. Chocho, Proc. of the $2^{\text {nd }}$ Int. Conf. On Nitride Semicond., Tokushima, Japan, October, 1997.

12. S. Nakamura, M.Senoh, S. Nagahama, N. Iwasa, T. Yamanda, T. Matsushita, H. Kiyoku, Y. Sugimoto, T. Kozaki, H. Umemoto, M. Sano, and K. Chocho, Appl. Phys. Lett., 72, 211 (1998).

13. S. Nakamura, M.Senoh, S. Nagahama, N. Iwasa, T. Yamanda, T. Matsushita, H. Kiyoku, Y. Sugimoto, T. Kozaki, H. Umemoto, M. Sano, and K. Chocho, Jpn. J. Appl. Phys., Part 2, 37 (1998), p. L309.

14. T. Zheleva, S. Smith, D. Thomson, K. Linthicum, T. Gerhke, P. Rajagopal, R.Davis, J. Electron. Matls. 28, L5 (1999)

15. K. J. Linthicum, T. Gehrke, D.Thomson, E. Carlson, P. Rajagopal, T.Smith, R. Davis, Appl. Phys Lett. 75, 196 (1999).

16. T. Gehrke, K. J. Linthicum, D.B.Thomson, P. Rajagopal, A. D. Batchelor and R. F. Davis, MRS Internet J. Nitride Semicond. Res. 4S1, G3.2 (1999).

17. K. J. Linthicum, T. Gehrke, D.B.Thomson, K. M. Tracy, E. P. Carlson, T. P. Smith, T. S. Zheleva, C. A. Zorman, M. Mechregany and R. F. Davis, MRS Internet J. Nitride Semicond. Res. 4S1, G4.9 (1999).

18. D.B.Thomson, T. Gehrke, K. J. Linthicum, P. Rajagopal, P. Hartlieb, T. S. Zheleva and R. F. Davis, MRS Internet J. Nitride Semicond. Res. 4S1, G3.37 (1999).

19. T. S. Zheleva, D.B.Thomson, S. Smith, P. Rajagopal, K. J. Linthicum, T. Gehrke, and R. F. Davis, MRS Internet J. Nitride Semicond. Res. 4S1, G3.36 (1999).

20. T. Weeks, M. Bremser, K. Ailey, E. Carlson, W. Perry, and R. Davis, Appl. Phys. Lett., 67, 401 (1995).

21. C. A. Zorman, A. J. Fleischman, A. S. Dawa, M. Mehregany, C. Jacob, S. Nishino and P. Pirouz, J. Appl. Phys. 78, 5136 (1995).

22. T. Gehrke, K.J. Linthicum, P. Rajagopal, E.A. Preble, E.P. Carlson, B.M. Robin, R.F. Davis, Int. Conf. on SiC and Related Materials (ICSCRM), Raleigh, NC, paper \#269 (1999). 